\title{
Unique Biosynthetic Pathway in Bloom-Forming Cyanobacterial Genus Microcystis Jointly Assembles Cytotoxic Aeruginoguanidines and Microguanidines
}

\author{
Pancrace Claire ${ }^{1,2}$, Ishida Keishi ${ }^{3}$, Briand Enora ${ }^{4}$, Pichi Douglas Gatte ${ }^{5}$, Weiz Annika R. ${ }^{5}$, \\ Guljarnow Arthur ${ }^{5}$, Scalvenzi Thibault ${ }^{1}$, Sassoon Nathalie ${ }^{1}$, Hertweck Christian ${ }^{3,6}$, Dittmann Elke ${ }^{5,{ }^{*} \text {, }}$ \\ Gugger Muriel ${ }^{1,}$
}

1 Inst Pasteur, Collect Cyanobacteries, 28 Rue Dr Roux, F-75724 Paris 15, France.

2 UPMC, UPEC, CNRS 7618, IEES Paris,UMR 113,IRD 242,INRA 1392,PARIS 7 113, 4 PI Jussieu, F75005 Paris, France.

${ }^{3}$ Hans Knoell Inst, Leibniz Inst Nat Prod Res \& Infect Biol, Beutenbergstr 11a, D-07745 Jena, Germany.

${ }^{4}$ IFREMER, Lab Phycotoxines, Rue lle Yeu, F-44311 Nantes, France.

${ }^{5}$ Univ Potsdam, Inst Biochem \& Biol, Dept Microbiol, D-14476 Golm, Germany.

${ }^{6}$ Friedrich Schiller Univ Jena, Fac Biol Sci, D-07743 Jena, Germany.

* Corresponding authors : Elke Dittmann, email address : editt@uni-potsdam.de ; Muriel Gugger, email address : $\underline{\text { muriel.gugger@pasteur.fr }}$

\begin{abstract}
:
The cyanobacterial genus Microcystis is known to produce an elaborate array of structurally unique and biologically active natural products, including hazardous cyanotoxins. Cytotoxic aeruginoguanidines represent a yet unexplored family of peptides featuring a trisubstituted benzene unit and farnesylated arginine derivatives. In this study, we aimed at assigning these compounds to a biosynthetic gene cluster by utilizing biosynthetic attributes deduced from public genomes of Microcystis and the sporadic distribution of the metabolite in axenic strains of the Pasteur Culture Collection of Cyanobacteria. By integrating genome mining with untargeted metabolomics using liquid chromatography with mass spectrometry, we linked aeruginoguanidine (AGD) to a nonribosomal peptide synthetase gene cluster and coassigned a significantly smaller product to this pathway, microguanidine (MGD), previously only reported from two Microcystis blooms. Further, a new intermediate class of compounds named microguanidine amides was uncovered, thereby further enlarging this compound family. The comparison of structurally divergent AGDs and MGDs reveals an outstanding versatility of this biosynthetic pathway and provides insights into the assembly of the two compound subfamilies. Strikingly, aeruginoguanidines and microguanidines were found to be as widespread as the hepatotoxic microcystins, but the occurrence of both toxin families appeared to be mutually exclusive.
\end{abstract}




\section{Graphical abstract :}

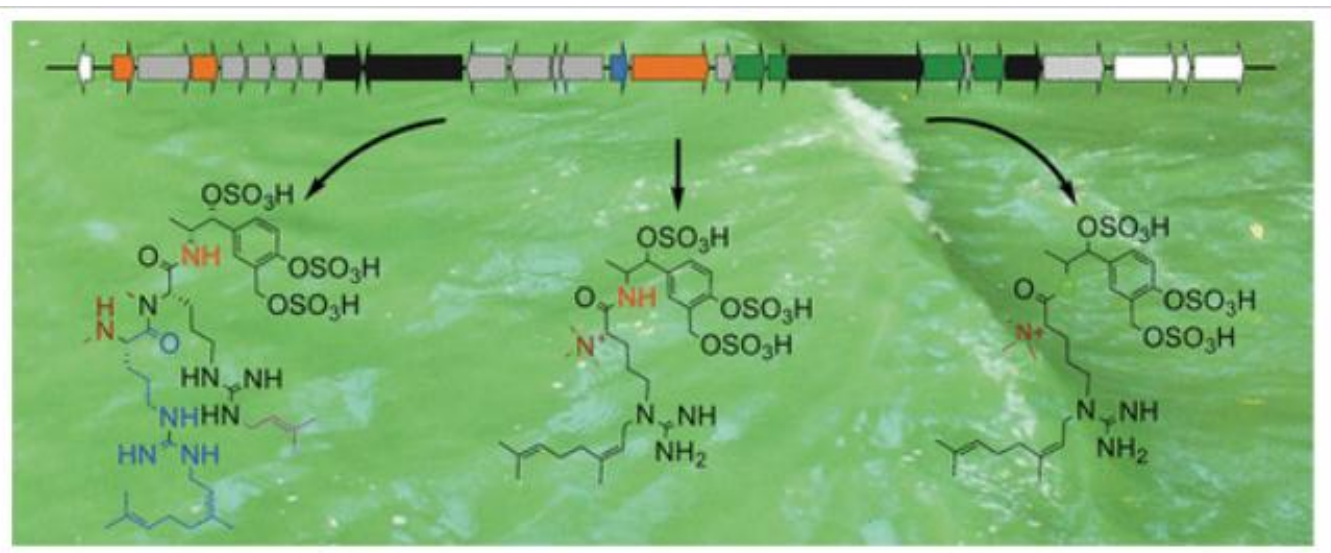


49

50

\section{INTRODUCTION}

Microcystis is a dominant bloom-forming cyanobacterium occurring in temperate freshwater ecosystems. ${ }^{1}$ The genus is infamous for the production of the well-known hepatotoxin microcystin. ${ }^{2}$ Both blooms and toxins cause ecosystem disturbance and public health threats, and constitute a growing concern in the frame of freshwater eutrophication and global warming. Microcystis has also been described as a producer of a multitude of bioactive natural products, some of interest for biotechnological and pharmaceutical application..$^{3-5}$

Cytotoxic aeruginoguanidines (AGDs) represent one of the most remarkable families of compounds described for Microcystis. ${ }^{6}$ The three AGD congeners reported for strain $M$. aeruginosa NIES-98 feature highly unprecedented characteristics such as a 1-(4hydroxy-3-hydroxymethyl)-phenyl-1-hydroxy-2-propylamine-4',3',1-tri- $O$-sulfate

(Hphpa trisulfate) moiety, along with geranylation and prenylation of arginines (Fig. 1A). While bloom-forming Microcystis belong to the most intensively studied cyanobacteria, AGDs were reported only twice from a bloom in Czech Republic and an isolate in Brazil,7, 8 and never from any other cyanobacteria. Their intricate features confine AGDs into a unique compound family. ${ }^{3}$

Our recent genomic analysis of ten Microcystis strains revealed that the different genotypes share a highly similar core genome while their biosynthetic gene clusters (BGCs) involved in natural product (NP) formation show a sporadic distribution. Moreover, we uncovered three cryptic BGCs not associated with any cyanobacterial compound. ${ }^{9}$ The continuously increasing number of publically available genomes of Microcystis further corroborates the high genetic diversity and patchy distribution of the NPs produced by this cyanobacterium.

Analysis of mass spectrometry (MS) data has been widely used for years in NP characterization efforts. Molecular networking computational approach uses tandem MS/MS data to group spectra based on their fragmentation patterns similarities, which gain strength in the frame of multi-strain comparison. Approaches combining molecular networking with genome mining highlight putative links between parent ions and pathways responsible for their biosynthesis. This combinatorial approach has been shown effective at linking NPs to their biosynthetic gene clusters in cyanobacteria and other prokaryotes such as Salinospora. ${ }^{10,11 .}$

Here, we have utilized the sporadic distribution of BGCs in Microcystis to assign one of 
82 the orphan BGCs to AGD. By integrating the genome sequence of the known AGDproducing strain Microcystis aeruginosa NIES-98,12 we screened Microcystis public genomes and axenic PCC strains for the AGD and its candidate BGC using genome mining, PCR and untargeted metabolomics. These data were further combined with molecular networking and genome comparison to link AGD to its biosynthetic gene cluster and study its diversity at the genetic and the metabolite level. The integrative approach allowed to enlarge the AGD compound family with microguanidine amide congeners (MGAs) and new variants of microguanidines (MGDs), and provides comprehensive insights into the extraordinary versatility of this biosynthetic pathway.

\section{RESULTS AND DISCUSSION}

93 Candidate synthesis BGC for sulfated, geranylated and prenylated compounds.

94 Considering the chemical structure of aeruginoguanidine (Figure 1A), the BGC involved 95 in its synthesis was expected to encode nonribosomal peptide synthetase (NRPS) 96 modules with specificity for L-arginine and tailoring enzymes such as a 97 prenyltransferase and a sulfatase/sulfotransferase. The genome of the AGD-producing 98 strain Microcystis aeruginosa NIES-98 contained only one cluster with these features, 99 which was homologous to the MIC2 cluster previously described in the genomes of 100 Microcystis aeruginosa PCC 9806 and PCC 9717 and Microcystis sp. T1-4. ${ }^{9}$ The candidate 101 BGC encoded two mono-modular NRPS, one of which comprising an integrated $\mathrm{N}$ 102 methylation domain as anticipated for the $N$-methylation of the Arg moieties. Substrate 103 prediction of the second NRPS was more ambiguous without excluding Arg (Table 1). 104 The putative AGD BGC, which spans $\sim 34 \mathrm{~kb}$ in the genome of Microcystis aeruginosa 105 NIES-98, includes 25 genes (Table 1) organized in three operons (Figure 1B). The two 106 NRPS AgdE and AgdK are accompanied by a predicted hydroxybenzoate synthase $107(\mathrm{AgdH})$, an AMP-dependent-ligase $(\operatorname{AgdA})$, a peptidyl carrier protein $(\mathrm{AgdB})$, a radical 108 SAM protein with decarboxylase function (AgdC) and two thioester reductases (AgdN 109 and AgdU). Several proteins consistent with tailoring enzymes involved in AGD 110 biosynthetic pathways are present such as two methyltransferases (AgdI, AgdM), an 111 aminotransferase (AgdL), an isoprenyltransferase (AgdJ), several 112 sulfatase/sulfotransferases (AgdD, AgdG, AgdP and AgdR), plus putative 113 permease/transporters (AgdF, AgdO), and thiamine pyrophosphatase (AgdQ) genes.

114 This candidate BGC for AGD present in seven genomes, including the public ones of 
115 Microcystis aeruginosa TAIHU98, Microcystis sp. SPC777 and CACIAM03, was used to 116 optimize specific primers and PCR conditions to detect its presence in Microcystis 117 strains. The two primer pairs designed were targeting two genes of the candidate BGC 118 presumably involved in an early and a late stage of AGD biosynthesis. Both genes do not 119 share homologies with other NRPS BGCs in Microcystis (agdH and agdJ, Table S1). The 120 screening of these two selected genes revealed seven additional PCC Microcystis strains, 121 whose on-going genome sequences helped to better define the limits of this BGC (Table 122 S2). A close inspection of the 14 genomes revealed the candidate AGD BGC with 28 genes 123 in perfect synteny, without rearrangement, and expanded the initial MIC2 cluster with 124 conserved neighboring genes (Figure 1B). Noteworthy, the largest NRPS gene agdK of 125 Microcystis sp. PCC 10613 was reduced to a remnant fragment, as confirmed by PCR. In 126 addition, the gene agdK was split in two in the genomes of Microcystis sp. CACIAM03 and 127 TAIHU98. Similarly, the gene agdQ was split in the genome of PCC 9624, while a contig 128 border separated $a g d P$ and $a g d Q$ in the genomes of PCC 9624 and PCC 10613. The 129 predicted aminotransferase gene $a g d L$ was lacking in the genomes of PCC 9717 and PCC 130 9810, also confirmed by PCR. Finally, the genes agdS and agdT, without known function, 131 appeared duplicated in ten strains (Figure 1B).

AGD and co-assignment of microguanidine by Molecular Networking. Detection of 134 AGD was performed by LC-MS/MS to assess its presence in the AGD producer strain 135 NIES-98 and in ten strains of the PCC containing the candidate BGC, as well as in eight 136 PCC strains that did not contain it in their genomes. Two molecular networks (MNs) 137 were constructed from LC-MS/MS data, one in positive mode $(\mathrm{MN}(+))$ and another in 138 negative mode $(\mathrm{MN}(-))$. In order to dereplicate the complex dataset, signatures of NPs 139 previously found in some of these Microcystis strains were identified using high140 performance liquid chromatography electrospray ionization mass spectrometry (HPLC141 ESI-MS/MS). Specifically, MS/MS fragments were identified for the cyanopeptolins A, B 142 and C in PCC 7806, aeruginosamides B and C and ferintoic acid (anabaenopeptins) in 143 PCC 9432, and ferintoic acid in PCC 9701 as predicted from their genomes (Figure S1A). 1449,13 The $\mathrm{MN}(+)$, consisting of 1998 nodes, was thus reliable in finding the expected 145 compounds. However, AGD was spread in several nodes of the $\mathrm{MN}(+)$ apart from each 146 other. Indeed, AGD had a better fragmentation pattern in negative mode as it was 147 collapsed into a single large node among the 1876 nodes of the MN(-) (Figure S1B). An 
148 extraction of the AGD node in $\mathrm{MN}(-)$ encompassed all strains carrying the full candidate 149 BGC for AGD synthesis, but neither the strain PCC 10613 nor the strains lacking this AGD 150 candidate cluster (Figure 2A). Up to 20 different putative variants of AGD were found in 151 these Microcystis strains, with strains NIES-98, PCC 9804, PCC 9805 and T1-4 able to 152 produce the three known AGD standards, whereas the other strains produced one or 153 two of those variants (Figure 2).

154 Strikingly, the $\mathrm{MN}(-)$ revealed that all the strains containing the AGD candidate BGC 155 produced also a significantly smaller product of $772 \mathrm{Da}$ (Figure 2B). Literature research 156 revealed that a compound with this mass, microguanidine AL772, was previously 157 reported for a Microcystis bloom. ${ }^{14}$ Microguanidines (MGDs) share striking similarities 158 with AGDs but display also considerable differences. Instead of the highly unusual 159 Hphpa trisulfate moiety, MGDs contain 3-(4-hydroxy-3-hydroxymethylphenyl)-2160 hydroxy-1-propanol (Hphpol). Further, MGDs feature a permethylation at the $\alpha$-amino 161 group of Arg that has not been observed in AGDs. Along with MGD AL772 (4, Figure 3, 162 related Figures S3 and S4A), a new MGD variant, MGD-704 (5, Figure 3, related Figures 163 S3 and S4B, and Table S3) was detected in the majority of strains differing from the two 164 other characterized MGDs, KT636 and DA368. ${ }^{14-16}$

165 In addition, the structural elucidation of the MGD size range compounds by MS 166 fragmentation and high-resolution MS analyses uncovered a novel intermediate class of 167 metabolites mixing features of AGD and MGD. While both compounds contain the Hphpa 168 trisulfate moiety linked with an amide bond to the arginine derivative as in AGDs they 169 were lacking the second arginine moiety and carried the same permethylation at the $\alpha$ 170 amino group of Arg as in MGDs (6 and 7, Figure 3, related Figures S3 and S4C and D, and 171 Table S3). To confirm the structure of $\mathbf{6}$, several 4 and $\mathbf{6}$ producing strains were 172 extracted and small amounts of $\mathbf{4}$ and $\mathbf{6}$ were purified by reversed-phase HPLC. The ${ }^{1} \mathrm{H}$ 173 NMR spectra of AGD 98-A (1), AGD 98-B (2), AGD 98-C (3), MGD AL772 (4), and MGA 174 (6) showed highly similar signals (Figure S5-S14). Detailed comparison of ${ }^{1} \mathrm{H}$ NMR 175 signals between 4 and 6 revealed three notable differences, namely the appearance of 176 new amide proton $\delta 8.48$ (H11 in 6), 1.02 and 0.18 ppm and high field shifted methine 177 protons H8 $\delta 5.21$ (4) to $\delta 4.23$ (6) and H13 $\delta 4.11$ (4) to $\delta 3.93$ (6), respectively (Figure 178 S5). The ${ }^{1} \mathrm{H}^{-1} \mathrm{H}$ COSY correlation from H8 to H11 and HSQC analysis of 6 indicated that $179 \mathrm{C} 8(\delta 49.8$ in $\mathbf{6}, \delta 75.3$ in $\mathbf{4})$ is adjacent to nitrogen (Figure S15-S18, Table S4). These 180 results strongly supported that the predicted structure of $\mathbf{6}$ indeed possesses an amide 
181 bond instead of the ester bond in 4 . As the low amount of 6 did not enable a sufficient 182 quality of ${ }^{13} \mathrm{C}$ NMR and other 2D NMR spectra, chemical shift assignment of 6 was 183 performed by the comparison with NMR data of 4. The stereochemistry of the geranyl 184 group of 6 was determined as $Z$-form, judging from the close similarity of chemical shifts 185 with 1-3 and ${ }^{13} \mathrm{C}$ NMR data of geraniol ( $E$-form) and nerol ( $Z$-form) 186 (www.chemicalbook.com/). This result further revealed that the stereochemistry of 187 geranyl group of MGD AL772 (4) also has Z-form. The new intermediate class of 188 compounds was designated microguanidine amide, with MGA-771 and MGA-787.

189 Indeed, the MGA peptides and the two MGD depsipeptides were observed 190 simultaneously with AGDs in four strains (PCC 9804, PCC 9805, PCC 9811 and T1-4). 191 Thus, Microcystis harboring the Agd BGC may build two different condensations 192 between the modified Arg residue and the phenetylalcohol (ester bond) in MGD 193 congeners or the phenetylamine (amide bond) in all AGD congeners (Figure 3).

194 The co-existence of AGD and MGD in the majority of Agd BGC positive strains, the 195 existence of a new intermediate class and the large overlap in anticipated biosynthetic 196 features lead us to conclude that AGD and MGD represent alternative products of the 197 same biosynthetic pathway. Remarkably, strain PCC 10613 lacking the NRPS gene agdK 198 was found to produce the MGDs in the MN(-) (Figure 2). Noteworthy, strain PCC 9624 in 199 which the Agd BGC differed at the level of the agdQ produced only the AGD-98A and the 200 MGD-AL772. Similarly, PCC 9810, PCC 9811 and PCC 9717 that lack the predicted 201 aminotransferase $a g d L$ and several Agd genes of unknown function (agdS', agdT') 202 produced a lower diversity of AGD variants under the same growing conditions than 203 other AGD producing Microcystis strains. None of the other Microcystis strains analyzed, 204 notably the ones containing the Mcy gene cluster, produced AGD, MGA or MGD. 205

206 Characterization of the BGC potentially involved in the AGD/MGD synthesis. One of 207 the most striking findings of our study is the extraordinary diversity of products 208 concurrently generated by the AGD/MGD pathway in single strains. Considering the 209 variations detected even in the backbone of AGDs and MGDs and in the linkage of their 210 individual moieties, the biosynthesis pathway cannot be considered as a classic 211 assembly line of NRPS. This pathway is rather a toolkit of enzymes optionally producing 212 a cocktail of metabolites that share the same precursors and similar tailoring 213 modifications but combine the different building blocks to alternative products. At the 
214 same time, the unprecedented diversity of products and intermediates and the existence 215 of natural mutants lacking individual biosynthetic genes allows for conclusions 216 regarding a number of biosynthetic steps of the complex pathway.

217 The presence of a putative $p$-hydroxybenzoate synthase $(\mathrm{AgdH})$ in the AGD cluster 218 indicates that the trisubstituted benzene unit of Hphpa and Hphpol might be derived 219 from chorismate ${ }^{17}$. Given that Hphpa and Hphpol possess a rare $m$-hydromethyl residue 220 in the benzene ring, AgdH might act in a similar way as isochorismate mutase, which has 221 been reported to catalyze the transformation of isochorismate to $m$ 222 carboxyphenylpyruvate. ${ }^{18,} 19$ We cannot dissect all individual steps towards the Hphpa 223 and Hphpol moieties, but we propose that the AMP-dependent ligase AgdA might 224 activate the $o$-carboxylic acid group of a $p$-hydroxyphenylpyruvate intermediate 225 followed by the transfer to the free-standing PCP AgdB (Figure 4). The resulting 226 thioester is presumably reduced to the corresponding alcohol either by thioester 227 reductase AgdN or $U$ through reductive chain termination as shown for myxochelin 228 biosynthesis in Stigmatella aurantiaca. ${ }^{20}$ A yet unassigned hydroxylation step at the $\beta$ 229 position of the $m$-hydroxymethyl- $p$-hydroxyphenylpyruvate yields 3-hydroxy- $m$ 230 hydroxymethyl-p-hydroxyphenylpyruvate as the precursor of both Hphpa and Hphpol. 231 We hypothesize that this precursor represents a branching point where further 232 transformation of the $\alpha$-keto group by aminotransferase AgdL yields Hphpa, while 233 transformation by a reductase (e.g. AgdN or U) yields Hphpol (Figure 4). This hypothesis 234 is supported by the fact that the lack of agdL in strains PCC 9717 and PCC 9810 still 235 permits production of MGD variants containing the Hphpol moiety (4 and 5) but not the 236 alternative Hphpa moiety as in MGAs (6 and 7). It is of note, that some of the predicted 237 biosynthetic steps for Hphpa and Hphpol biosynthesis (Figure 4) share similarities to 238 enzyme reactions involved in biosynthesis of the characteristic Choi moiety in the 239 aeruginosin pathway ${ }^{21}$. In this context, it is worth mentioning that the majority of 240 AGD/MGD producers also harbor aeruginosin biosynthesis genes in their genome 241 (Figure 5), thus not excluding the possibility of a joint use of precursors and enzymes.

242 Furthermore, the strain M. aeruginosa PCC 10613 can be considered as a natural agdK 243 mutant, thus allowing deducing the roles of the two NRPSs in the pathway. The fact that 244 the lack of AgdK in PCC 10613 still enables MGD production strongly suggests that AgdE 245 is the responsible NRPS activating Arg in the MGD and MGA pathways (Figure 6). On the 246 other hand, the NRPS AgdK harbouring an N-methyltransferase domain is likely 
247 incorporating $N$-Me-Arg in the AGD pathway. Whether or not AgdK acts iteratively or 248 cooperates with AgdE to yield the MeArg-MeArg-Hphpa moiety of AGDs cannot be 249 dissected based on the current dataset. The biosynthetic intermediate(s) might be 250 methylated and decarboxylated by the radical SAM enzyme AgdC. Since AgdC shows 251 close homology to the oxygen-independent coproporphyrinogen III oxidase of E.coli $252(\mathrm{HemN})$ we propose that it utilizes a 5-deoxyadenosyl radical to trigger a 253 decarboxylation reaction as demonstrated for the HemN enzyme family. ${ }^{22}$ The 254 intermediate may further be modified by several tailoring enzymatic reactions such as $255 N$-methylation (methyltransferase; AgdI or $\mathrm{M}$ ) of Arg residue, to the tri-sulfation 256 (sulfotransferases; AgdD, P and R, sulfatase; AgdG) of the Hphpa residue, and the $N$ 257 alkylation (isoprenyltransferase; AgdJ) of $N$-MeArg residues. Some of the proposed 258 biosynthetic steps may occur while substrates are tethered on PCP-domains of NRPSs or 259 the standalone peptidyl carrier protein AgdB. The fact that no desulfated intermediates 260 were observed in the MS/MS networking may suggest that sulfation of the aromatic 261 moiety occurs in the PCP-bound state.

262 The distinct alkylation pattern at the guanidinyl group of $N$-trimethyl Arg ( $\omega$ for AGDs 263 and $\varepsilon$ for MGDs) may derive from alternative substrate specificities of the 264 isoprenyltransferase AgdJ (Figure 6). Comparison of the distinct AGD/MGD product 265 profiles of individual Microcystis strains thus suggests an outstanding versatility of the 266 pathway. A complete assignment of biosynthetic steps will require biochemical 267 characterization of participating enzymes and targeted feeding studies, yet the analysis 268 of natural agdK and agdL mutants led to definite conclusions regarding the role of these 269 two enzymes.

270 The example of the joint AGD/MGD pathway further strengthens the paradigm that 271 cyanobacteria have evolved unique mechanisms to produce diverse NPs of high 272 complexity in single strains using limited genetic resources. Other cyanobacterial 273 mechanisms include the utilization of alternative starter modules for NRPS as shown for 274 the anabaenopeptin synthetase of strain Anabaena 90,23 the integration of multispecific 275 adenylation domains of NRPS as shown for the anabaenopeptin synthetase of 276 Planktothrix NIVA-CYA 126, ${ }^{24}$ and the microcystin synthetase in Microcystis aeruginosa 277 NIES 843. ${ }^{25}$ Recently, a simultaneous production of anabaenopeptins and namalides 278 allowed to reveal a single pathway for their synthesis. ${ }^{26}$ We can only speculate whether 
279 AGDs and MGDs act synergistically or fulfill parallel independent functions in the 280 producing strains.

281 An interesting phenomenon observed during this study is that AGD/MGD production 282 and MC production are almost mutually exclusive among Microcystis strains. The only 283 exception was found in the genomes of two non-monoclonal Brazilian strains, ${ }^{27,} 28$ that 284 carry both clusters and for which the production of these compounds is not yet 285 documented. There is increasing evidence that MCs are closely interfering with the 286 primary metabolism of Microcystis in addition to their toxicity. ${ }^{29}$ Whether or not AGD 287 and MGD can complement for the loss of MC or reflect a different niche adaptation of 288 their respective producers remains elusive.

289 Our study further suggests that the rare detection of AGD and MGD in only two 290 Microcystis aeruginosa isolated in Japan and in Brazil (NIES $98^{6}$ and NPCD-18) and bloom 291 materials of Microcystis in Israel ${ }^{14-16}$ respectively is not due to the scarce occurrence of 292 these metabolites among Microcystis, but rather to the lack of attention towards these 293 peculiar NPs in previous studies. Thus, the AGD/MGD producers seem to be as dispersed 294 worldwide as the MC producing strains, and therefore should be considered in future 295 screening of Microcystis blooms and isolates.

\section{CONCLUSIONS}

298 Cyanobacteria are infamous for worldwide bloom formation in freshwater bodies. Risk 299 assessment of Microcystis blooms primarily considers the hepatotoxin microcystin (MC). 300 The present study suggests that the neglected family of compounds, cytotoxic 301 aeruginoguanidines and microguanidines, is more frequently produced than previously 302 anticipated, mainly in non-MC producing Microcystis strains. Remarkably, the two 303 structurally divergent groups of compounds are products of a branched and versatile 304 biosynthetic pathway. The genetically constraint gene cluster generates a library of 305 diverse products in single strains and further strengthens the paradigm that 306 cyanobacteria have developed unique mechanisms to generate metabolic diversity. 307 These findings open new perspectives for future studies on orphan natural products and 308 evolution of their biosynthetic pathways. 


\section{MATERIALS AND METHODS}

313 Strain cultures and detection of the cluster. Axenic Microcystis strains from the PCC 314 and from the NIES collections were grown at $25{ }^{\circ} \mathrm{C}$ in $40 \mathrm{~mL} \mathrm{BG11_{0 }}$ medium $^{30}$ 315 supplemented with $2 \mathrm{mM} \mathrm{NaNO}_{3}$ and $10 \mathrm{mM} \mathrm{NaHCO}_{3}$ under continuous light (Table S2). 316 For nucleic acid extraction, chemical and PCR analysis, the details are described in 317 Supporting information.

318 Sequencing \& genomics analysis. For the strains suspected to carry the agd gene 319 cluster, whole genome sequencing was performed by the Mutualized Platform for 320 Microbiology at Institut Pasteur. Genomes were integrated in the MicroScope platform ${ }^{31}$ 321 for further analysis. The genome sequencing is described in Supplemental information. 322 The species tree was generated by a concatenation of 586 conserved proteins selected 323 from the phylogenetic markers previously validated for Cyanobacteria. ${ }^{32}$ Phylogenetic 324 analysis is detailed in Supplemental information. AntiSMASH 3.0 33 was used to identify 325 the targeted BGC in each genome sequence. In cases where the agd gene cluster spanned 326 several contigs/scaffolds PCRs were performed to confirm the colocalization of the gene 327 cluster parts in the same genomic locus (Table S1).

328 Cyanobacterial cell extraction. Lyophilized cyanobacterial cells from $200 \mathrm{~mL}$ cultures 329 of 19 Microcystis aeruginosa strains were extracted with $80 \%$ aqueous methanol (v/v, $33025 \mathrm{~mL}$ ) using a sonicator (Sonoplus MS73, Bandelin, 30\% power, 5 cycles for 2 min at 331 room temperature). Each extract was centrifuged at 8,000 $\times$ g for $15 \mathrm{~min}$ at $15{ }^{\circ} \mathrm{C}$. The 332 residues were extracted with $80 \%$ aqueous methanol (v/v, $25 \mathrm{~mL}$ ) and methanol (25 $333 \mathrm{~mL}$ ), respectively, as the above-mentioned procedure. The extracts were combined and 334 dried under a reduced pressure. The crude residues were dissolved in $50 \%$ aqueous 335 methanol $(\mathrm{v} / \mathrm{v}, 1 \mathrm{~mL})$ and kept in a fridge until analysis.

336 HPLC-MS measurement. LC-MS/MS measurements were carried out by Bruker HCT 337 Ultra ion trap mass spectrometry (BrukerDaltonics, Bremen, Germany) coupled with an 338 Agilent Technologies 1100 series liquid chromatogram system (Agilent, Waldbronn, 339 Germany). The HR-LCMS measurements were performed by HPLC-HRMS series of 340 Thermo Accela (LC) and Thermo Exactive (HRMS), an ESI source operating in both 341 polarity mode and an orbitrap analyzer (Thermo Fisher Scientific, Bremen). The details 342 of both measurements are described in Supporting Information.

343 Molecular networking. LC-MS/MS data acquired from Bruker instrument were used 344 for molecular networking. Two molecular networks (MNs) were performed with LC- 
345 MS/MS data, one in positive mode $(\mathrm{MN}(+))$ and another with negative mode data $(\mathrm{MN}(-$ 346 J) with LC-MS/MS data from Microcystis strains and AGD A, B and C standards. The steps 347 followed for both MNs are described in Supporting Information.

\section{REFERENCES}

350

351

352

353

354

355

356

357

358

359

360

361

362

363

364

365

366

367

368

369

370

371

372

373

374

375

376

1. Harke, M. J., Steffen, M. M., Gobler, C. J., Otten, T. G., Wilhelm, S. W., Wood, S. A., and Paerl, H. W. (2016) A review of the global ecology, genomics, and biogeography of the toxic cyanobacterium, Microcystis spp, Harmful Algae 54, 4-20.

2. Merel, S., Walker, D., Chicana, R., Snyder, S., Baures, E., and Thomas, O. (2013) State of knowledge and concerns on cyanobacterial blooms and cyanotoxins, Environ. Int. 59, $303-327$.

3. Welker, M., and von Dohren, H. (2006) Cyanobacterial peptides - nature's own combinatorial biosynthesis, FEMS Microbiol. Rev. 30, 530-563.

4. Kehr, J. C., Gatte Picchi, D., and Dittmann, E. (2011) Natural product biosyntheses in cyanobacteria: A treasure trove of unique enzymes, Beilstein J. Org. Chem. 7, 16221635.

5. Dittmann, E., Gugger, M., Sivonen, K., and Fewer, D. P. (2015) Natural product biosynthetic diversity and comparative genomics of the Cyanobacteria, Trends Microbiol. 23, 642-652.

6. Ishida, K., Matsuda, H., Okita, Y., and Murakami, M. (2002) Aeruginoguanidines 98-A98-C: cytotoxic unusual peptides from the cyanobacterium Microcystis aeruginosa, Tetrahedron 58, 7645-7652.

7. Welker, M., Marsalek, B., Sejnohova, L., and von Dohren, H. (2006) Detection and identification of oligopeptides in Microcystis (cyanobacteria) colonies: Toward an understanding of metabolic diversity, Peptides 27, 2090-2103.

8. Silva-Stenico, M., da Silva, C., Lorenzi, A., Shishido, T., Etchegaray, A., Lira, S., Moraes, L., and Fiore, M. (2011) Non-ribosomal peptides produced by Brazilian cyanobacterial isolates with antimicrobial activity, Microbiol. Res. 166, 161-175.

9. Humbert, J. F., Barbe, V., Latifi, A., Gugger, M., Calteau, A., Coursin, T., Lajus, A., Castelli, V., Oztas, S., Samson, G., Longin, C., Medigue, C., and de Marsac, N. T. (2013) A tribute to disorder in the genome of the bloom-forming freshwater cyanobacterium Microcystis aeruginosa, PLoS One 8, e70747. 
377 10. Duncan, K. R., Crusemann, M., Lechner, A., Sarkar, A., Li, J., Ziemert, N., Wang, M., 378 Bandeira, N., Moore, B. S., Dorrestein, P. C., and Jensen, P. R. (2015) Molecular 379 networking and pattern-based genome mining improves discovery of biosynthetic 380 gene clusters and their products from Salinispora species, Chem. Biol. 22, 460-471.

381 11. Moss, N. A., Bertin, M. J., Kleigrewe, K., Leao, T. F., Gerwick, L., and Gerwick, W. H. 382 (2016) Integrating mass spectrometry and genomics for cyanobacterial metabolite 383 discovery, J. Ind. Microbiol. Biotechnol. 43, 313-324.

384

12. Yamaguchi, H., Suzuki, S., Sano, T., Tanabe, Y., Nakajima, N., and Kawachi, M. (2016) 385 386 Draft genome sequence of Microcystis aeruginosa NIES-98, a non-microcystinproducing cyanobacterium from Lake Kasumigaura, Japan, Genome Announc. 4, e01187-01116.

389

13. Briand, E., Bormans, M., Gugger, M., Dorrestein, P. C., and Gerwick, W. H. (2016) Changes in secondary metabolic profiles of Microcystis aeruginosa strains in 390 response to intraspecific interactions, Environ. Microbiol. 18, 384-400.

14. Gesner-Apter, S., and Carmeli, S. (2008) Three novel metabolites from a bloom of 392 cyanobacterium Microcystis sp., Tetrahedron 64, 6628-6634.

15. Adiv, S., and Carmeli, S. (2013) Protease inhibitors from Microcystis aeruginosa 394 395 bloom material collected from the Dalton Reservoir, Israel, J. Nat. Prod. 76, 23072315.

16. Lifshits, M., and Carmeli, S. (2012) Metabolites of Microcystis aeruginosa bloom 397 material from Lake Kinneret, Israel, J. Nat. Prod. 75, 209-219.

17. Siebert, M., Severin, K., and Heide, L. (1994) Formation of 4-hydroxybenzoate in Escherichia coli: Characterization of the ubiC gene and its encoded enzyme 400 401 chorismate pyruvate-lyase., Microbiology 140, 897-904.

18. Zamir, L. O., Nikolokakis, A., Bonner, C. A., and Jensen, R. A. (1993) Evidence for 402 enzymatic formation of isoprephenate from isochorismate, Bioorganic Med. Chem. 403 Lett. 3, 1441-1446.

19. Blasiak, L. C., and Clardy, J. J. (2010) Discovery of 3-formyl-tyrosine metabolites from Pseudoalteromonas tunicata through heterologous expression, Am. Chem. Soc. 132, $926-927$.

20. Li, Y., Weissman, K., and Müller, R. (2008) Myxochelin biosynthesis: Direct evidence for two- and four-electron reduction of a carrier protein-bound thioester, J. Am. Chem. Soc. 130, 7554-7555. 
21. Ishida, K., Christiansen, G., Yoshida, W., Kurmayer, R., Welker, M., Valls, N., Bonjoch, J., Hertweck, C., Börner, T., Hemscheidt, T., and Dittmann, E. (2007) Biosynthesis and structure of aeruginoside $126 \mathrm{~A}$ and $126 \mathrm{~B}$, cyanobacterial peptide glycosides bearing a 2-carboxy-6-hydroxyoctahydroindole moiety, Chem. Biol. 14, 565-576.

22. Layer, G., Pierik, A. J., Trost, M., Rigby, S. E., Leech, H. K., Grage, K., Breckau, D., Astner, I., Jansch, L., Heathcote, P., Warren, M. J., Heinz, D. W., and Jahn, D. (2006) The substrate radical of Escherichia coli oxygen-independent coproporphyrinogen III oxidase HemN, J. Biol. Chem. 281, 15727-15734.

23. Rouhiainen, L., Jokela, J., Fewer, D. P., Urmann, M., and Sivonen, K. (2010) Two alternative starter modules for the non-ribosomal biosynthesis of specific anabaenopeptin variants in Anabaena (Cyanobacteria), Chem. Biol. 17, 265-273.

24. Kaljunen, H., Schiefelbein, S., Stummer, D., Kozak, S., Meijers, R., Christiansen, G., and Rentmeister, A. (2015) Structural elucidation of the bispecificity of A domains as a basis for activating non-natural amino acids, Angew. Chem. Int. Ed. Engl. 54, 88338836.

25. Meyer, S., Kehr, J., Mainz, A., Dehm, D., Petras, D., Süssmuth, R., and Dittmann, E. (2016) Biochemical dissection of the natural diversification of microcystin provides lessons for synthetic biology of NRPS, Cell Chem. Biol. 23, 462-471.

26. Shishido, T. K., Jokela, J., Fewer, D. P., Wahlsten, M., Fiore, M. F., and Sivonen, K. (2017) Simultaneous production of anabaenopeptins and namalides by the cyanobacterium Nostoc sp. CEN543, ACS Chem. Biol. 12, 2746-2755.

27. Fiore, M. F., Alvarenga, D. O., Varani, A. M., Hoff-Risseti, C., Crespim, E., Ramos, R. T., Silva, A., Schaker, P. D., Heck, K., Rigonato, J., and Schneider, M. P. (2013) Draft genome sequence of the Brazilian toxic bloom-forming cyanobacterium Microcystis aeruginosa strain SPC777, Genome Announc. 1, e00547-00513.

28. Castro, W. O., Lima, A. R., Moraes, P. H., Siqueira, A., Aguiar, D., Baraúna, A., Martins, L., Fuzii, H., de Lima, C., Vianez-Júnior, J., Nunes, M., Dall'Agnol, L., and Gonçalves, E. (2016) Draft genome sequence of Microcystis aeruginosa CACIAM 03, a cyanobacterium isolated from an Amazonian freshwater environment, Genome Announc. 4, e01299.

29. Neilan, B., Pearson, L., Muenchhoff, J., Moffitt, M., and Dittmann, E. (2013) Environmental conditions that influence toxin biosynthesis in cyanobacteria, Environ. Microbiol. 15, 1239-1253. 
443 30. Rippka, R., Deruelles, J., Waterbury, J. B., Herdman, M., and Stanier, R. Y. (1979) 444 Generic assignments, strain histories and properties of pure cultures of 445 Cyanobacteria, J. Gen. Microbiol. 111, 1-61.

446 31. Vallenet, D., Calteau, A., Cruveiller, S., Gachet, M., Lajus, A., Josso, A., Mercier, J., 447 Renaux, A., Rollin, J., Rouy, Z., Roche, D., Scarpelli, C., and Medigue, C. (2017) $448 \quad$ MicroScope in 2017: An expanding and evolving integrated resource for community 449 expertise of microbial genomes, Nucleic Acids Res. 45, D517-D528.

450

32. Pancrace, C., Barny, M., Ueoka, R., Calteau, A., Scalvenzi, T., Pedron, J., Barbe, V., Piel, J., 451 and Humbert, J. (2017) Insights into the Planktothrix genus: Genomic and metabolic comparison of benthic and planktic strains, Sci. Rep. 7, 41181.

453

33. Weber, T., Blin, K., Duddela, S., Krug, D., Kim, H. U., Bruccoleri, R., Lee, S. Y., Fischbach, 454 M. A., Muller, R., Wohlleben, W., Breitling, R., Takano, E., and Medema, M. H. (2015) antiSMASH 3.0-a comprehensive resource for the genome mining of biosynthetic 456 gene clusters, Nucleic Acids Res. 43, W237-243.

\section{FIGURE LEGENDS}

459 Figure 1. Aeruginoguanidines and the corresponding biosynthetic gene cluster. (A) The 460 structure of aeruginoguanidines (AGDs), 1; AGD-98A, 2; AGD-98B, 3; AGD-98C; (B) AGD 461 biosynthetic gene cluster of Microcystis aeruginosa NIES-98 and its variation in 13 other 462 Microcystis genomes sharing 94 to $98 \%$ of similarity. The genes are color-coded with 463 orange for carbohydrate sulfotransferase, sulfotransferase and sulfatase; black for NRPS 464 and thioesterase; green for methyl-, isoprenyl- and aminotransferase; blue for 465 permease; grey for proteins with putative and unknown function. The blue line indicates 466 the span of the MIC2 cluster previously described. ${ }^{9}$

467 Figure 2. Molecular network of AGD (A) and of MGD with MGA (B). Characterized 468 structural variants are indicated as red-colored nodes and new congeners characterized 469 in this study are highlighted in green. Diversity and distribution of AGD and MGD 470 variants for each strain. Details of $M N(+), M N(-)$, and the AGD and MGD networks are 471 presented in Figures S1-S3.

472 Figure 3. Microguanidine and microguanidine amide variants detected in strains 473 containing the AGD biosynthetic gene cluster. The MGD depsipeptides contain ester474 bonds, while the peptidic MGAs contain amide-bonds in their structures. Details of the 475 high-resolution MS data of MGA and MGD are presented in Table S3. 
476 Figure 4. Proposed Hphpa and Hphpol biosynthesis. 3-hydroxy-m-hydroxymethyl- $p$ 477 hydroxyphenylpyruvate is synthesized as a precursor of both Hphpa and Hphpol via 478 several steps from isochorismate. The resulting intermediate is further transformed by 479 either an aminotransferase (AgdL) or a reductase (AgdN or U) to yield Hphpa or Hphpol, 480 respectively and further transferred to the free-standing PCP (AgdB) after adenylation 481 by AgdA. The AgdL enzyme for which natural mutants were identified in the course of 482 this study is highlighted in red.

483 Figure 5. Distribution of the known and unknown BGCs in the frame of the phylogeny of 484 the 23 Microcystis genomes based on maximum likelihood tree built upon 586 marker 485 genes. The known BGCs are involved in the synthesis of aeruginoguanidine (AGD) and 486 microguanidine (MGD) and/or MGA only, of microcystin, of cyanobactins including 487 aeruginosamide, of aeruginosin, of microviridin, of cyanopeptolin, of anabaenopeptin 488 including ferintoic acid, and of microginin. One BGC only predicted in one strain is 489 involved in synthesis of puwainaphycin. ${ }^{12}$ The numbers indicate the unknown BGCs 490 detected in the genome; the origin of each strain is indicated in parenthesis.

491 Figure 6. Proposed AGD, MGA and MGD biosynthetic pathways. Top line; AGD 492 biosynthesis route: Hphpa which is linked to the free-standing PCP AgdB is transferred 493 to AgdE and condensed with the dipeptide, which is derived from AgdK and E. The 494 thioester-tethered intermediate is methylated by a radical SAM enzyme (AgdC) followed 495 by decarboxylation and released from the enzyme. The resulting molecule is further 496 modified by sulfation and farnesylation. Middle line: MGA (6 and 7) biosynthesis route, 497 almost the same pathway as AGD biosynthesis, but only AgdE is used and the $\alpha$-amino 498 group of Arg is permethylated by AgdI or M. Bottom line; MGD (4 and 5) biosynthesis 499 route, almost the same pathway as MGAs, but using Hphpol as the intermediate instead 500 of Hphpa. The enzyme AgdK for which a natural mutant was identified in the course of 501 this study is highlighted in red.

502

\section{TABLE}

504 Table 1. Proposed function of proteins encoded in the AGD gene cluster and flanking 505 ORFs in Microcystis aeruginosa NIES-98. The strand position and the size of gene in 506 amino acids are indicated with the corresponding Best BLASTp hit and identity, all 507 found in Microcystis genomes. NRPS domains: $\mathrm{C}$ for condensation, A for adenylation with 508 substrate prediction, PCP for peptidyl carrier protein, and nMT for N-methyl transferase. 
509

\section{ASSOCIATED CONTENT}

\section{Supporting Information}

512 The supporting Information is available free of charge via the ACS Publications

513 website at $\mathrm{DOI}$

514 Methods of preparation of the extracts and of recovering complete AGD cluster, HLPC-

515 MS measurement and molecular networking; four supporting tables and 18 supporting

516 figures on the detailed molecular network and the spectra of the new structures, as

517 indicated in the text (PDF).

518 Accession Codes

519 New sequence data are archived in GenBank under accession numbers $520 \mathrm{MH049490}$ to MH049500.

521

522 AUTHOR INFORMATION

523 Corresponding Author

$524 *$ email: muriel.gugger@pasteur.fr

$525 *$ email: editt@uni-potsdam.de

526 ORCID

527 Muriel Gugger: 0000-0001-6728-1976

528 Enora Briand: 0000-0001-8996-0072

529 Elke Dittmann: 0000-0002-7549-7918

530 Christian Hertweck: 0000-0002-0367-337X

531 Douglas Gatte Pichi: 0000-0001-9164-8969

532 Thibault Scalvenzi: 0000-0002-5760-1574

533

534 Notes

535 The author declare no competing financial interest

536

537 ACKNOWLEDGMENTS 
538 CP was supported by the Ile-de-France ARDoC Grant for PhD. Funding was 539 provided by the Institut Pasteur. ED was supported by a grant of the 540 German Research Foundation (DFG, Di910/10-1). Financial support by the 541 DFG-funded Collaborative Research Centre ChemBioSys (SFB 1127) to ED 542 and $\mathrm{CH}$ is gratefully acknowledged. We thank $\mathrm{A}$. Perner and $\mathrm{H}$. Heinecke for 543 Thermo Exactive LC-MS measurements. All PCC cyanobacteria of this study 544 are available from the Institut Pasteur. All data are contained in the main 545 text and supplementary materials. 
Table 1. Proposed function of proteins encoded in the AGD gene cluster and flanking ORFs in Microcystis aeruginosa NIES-98. The strand position and the size of gene in amino acids are indicated with the corresponding Best BLASTp hit and identity, all found in Microcystis genomes. NRPS domains: $C$ for condensation, $A$ for adenylation with substrate prediction, PCP for peptidyl carrier protein, and nMT for N-methyl transferase.

\begin{tabular}{|c|c|c|c|c|}
\hline $\begin{array}{l}\text { Gene } \\
\text { (Strand) }\end{array}$ & $\begin{array}{l}\text { Size } \\
\text { (aa) }\end{array}$ & $\begin{array}{l}\text { Proposed function (NRPS } \\
\text { with substrat prediction) }\end{array}$ & Best BLASTp hit (Accession number) & $\begin{array}{c}\text { Identity } \\
(\%)\end{array}$ \\
\hline Orf (-) & 160 & $\begin{array}{l}\text { Conserved protein of } \\
\text { unknown function }\end{array}$ & Hypothetical protein 053_4696 (ELP52967.1) & 100 \\
\hline $\operatorname{agdP}(+)$ & 238 & \begin{tabular}{|l|} 
Carbohydrate \\
sulfotransferase II
\end{tabular} & Hypothetical protein 053_4419 (ELP52967.1) & 100 \\
\hline agdQ (+) & 589 & $\begin{array}{l}\text { Thiamine pyrophosphate } \\
\text { enzyme }\end{array}$ & Acetolactate synthase large subunit (EPF22845.1) & 100 \\
\hline $\operatorname{agdR}(+)$ & 296 & Sulfotransferase I & Sulfotransferase domain protein (ELP52945.1) & 100 \\
\hline $\operatorname{agdS}(+)$ & 271 & $\begin{array}{l}\text { Conserved protein of } \\
\text { unknown function }\end{array}$ & Hypothetical protein 053_4433 (ELP52708.1) & 95 \\
\hline agdT (+) & 274 & $\begin{array}{l}\text { Conserved protein of } \\
\text { unknown function }\end{array}$ & Conserved hypothetical protein ( $\mathrm{CCH} 98454.1)$ & 99 \\
\hline agdS' (+) & 268 & $\begin{array}{l}\text { Conserved protein of } \\
\text { unknown function }\end{array}$ & Hypothetical protein MAESPC_01420 (EPF22841.1) & 99 \\
\hline agdT' (+) & 270 & $\begin{array}{l}\text { Conserved protein of } \\
\text { unknown function }\end{array}$ & $\begin{array}{l}\text { Putative uncharacterized ORF3 domain protein } \\
\text { (ELP52673.1) }\end{array}$ & 99 \\
\hline agdU (+) & 405 & Thioester reductase & Polyketide synthase hetM (CCI12982.1) & 98 \\
\hline $\operatorname{agdE}(-)$ & 1093 & 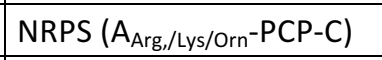 & Linear gramicidin synthase subunit D (EPF22838.1) & 98 \\
\hline $\operatorname{agdD}(-)$ & 441 & Sulfotransferase III & Zinc chelation protein SecC (WP_069474152.1) & 100 \\
\hline $\operatorname{agdC}(-)$ & 438 & Radical SAM & $\begin{array}{l}\text { Radical SAM superfamily protein (ELP52520.1) } \\
\text { putative oxygen-independent coproporphyrinogen III } \\
\text { synthase }\end{array}$ & 100 \\
\hline $\operatorname{agdB}(-)$ & 94 & Peptidyl carrier protein & $\begin{array}{l}\text { Phosphopantetheine attachment site family protein } \\
\text { (ELP52599.1) }\end{array}$ & 100 \\
\hline $\operatorname{agd} A(-)$ & 473 & $\begin{array}{l}\text { AMP-dependent } \\
\text { synthetase and ligase }\end{array}$ & AMP-dependent synthetase (WP_069474153.1) & 100 \\
\hline agdF (+) & 196 & Permease & Conserved hypothetical protein (CCI31673.1) & 97 \\
\hline agdG (+) & 852 & Sulfatase & Sulfatase family protein (ELP52537.1) & 99 \\
\hline agdH (+) & 191 & $\begin{array}{l}\text { 4-Hydroxybenzoate } \\
\text { synthetase }\end{array}$ & Hypothetical protein 053_4514 (ELP52787.1) & 100 \\
\hline agdl (+) & 342 & O-Methyltransferase & Methyltransferase (WP_069474155.1) & 100 \\
\hline agdJ $(+)$ & 231 & Isoprenyl-transferase & $\begin{array}{l}\text { Di-trans, poly-cis-decaprenylcistransferase } \\
\text { (ELP52925.1) }\end{array}$ & 99 \\
\hline $\operatorname{agdK}(+)$ & 1588 & NRPS $\left(\mathrm{A}_{\mathrm{Arg}}-\mathrm{nMT}-\mathrm{PCP}-\mathrm{C}\right)$ & Chondramide synthase cmdD (EPF22828.1) & 99 \\
\hline agdL (+) & 455 & Aminotransferase & Uncharacterized aminotransferase yodT (CCI31679.1) & 99 \\
\hline Orf (+) & 71 & Hypothetical protein & Hypothetical protein (WP_069474158.1) & 100 \\
\hline $\begin{array}{l}\text { agdM } \\
(+)\end{array}$ & 346 & O-Methyltransferase & O-Methyltransferase family protein (ELP53140.1) & 99 \\
\hline $\operatorname{agdN}(+)$ & 401 & Thioester reductase & Thioester reductase domain protein (ELP52682.1) & 99 \\
\hline agdo (+) & 671 & $A B C$ transporter & $\begin{array}{l}\text { ABC Transporter transmembrane region } 2 \text { family } \\
\text { protein (ELP52531.1) }\end{array}$ & 99 \\
\hline Orf (+) & 671 & $\begin{array}{l}\text { Conserved protein of } \\
\text { unknown function }\end{array}$ & Hypothetical protein 053_4447 (ELP52722.1) & 99 \\
\hline Orf (+) & 156 & $\begin{array}{l}\text { Conserved protein of } \\
\text { unknown function }\end{array}$ & Hypothetical protein 053_4299 (ELP52574.1) & 100 \\
\hline Orf (+) & 554 & GUN4-like family protein & Hypothetical protein (WP_069474163.1) & 100 \\
\hline
\end{tabular}


Figure 1. Aeruginoguanidines and the corresponding biosynthetic gene cluster. ( $A$ ) The structure of aeruginoguanidines (AGDs), 1; AGD-98A, 2; AGD-98B, 3; AGD-98C; (B) AGD biosynthetic gene cluster of Microcystis aeruginosa NIES-98 and its variation in 13 other Microcystis genomes sharing 94 to $98 \%$ of similarity. The genes are color-coded with orange for carbohydrate sulfotransferase, sulfotransferase and sulfatase; black for NRPS and thioesterase; green for methyl-, isoprenyl- and aminotransferase; blue for permease; grey for proteins with putative and unknown function. The dashed arrows under the cluster indicate the three operons. The blue line indicates the span of the MIC2 cluster previously described. ${ }^{9}$

A

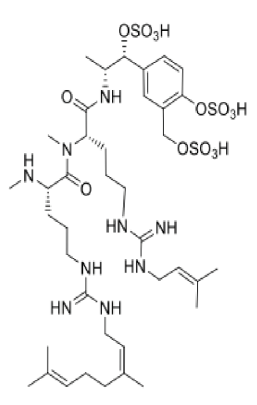

AGD-98A (1)

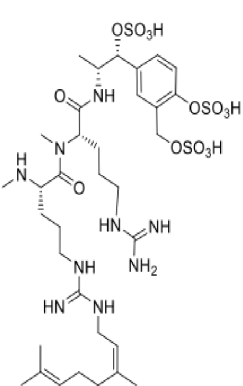

AGD-98B (2)

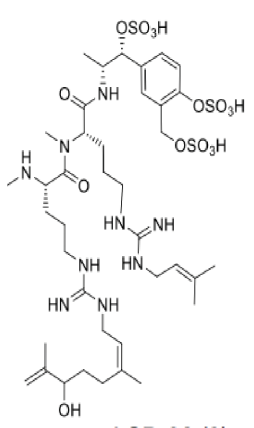

AGD-98 (3)

B

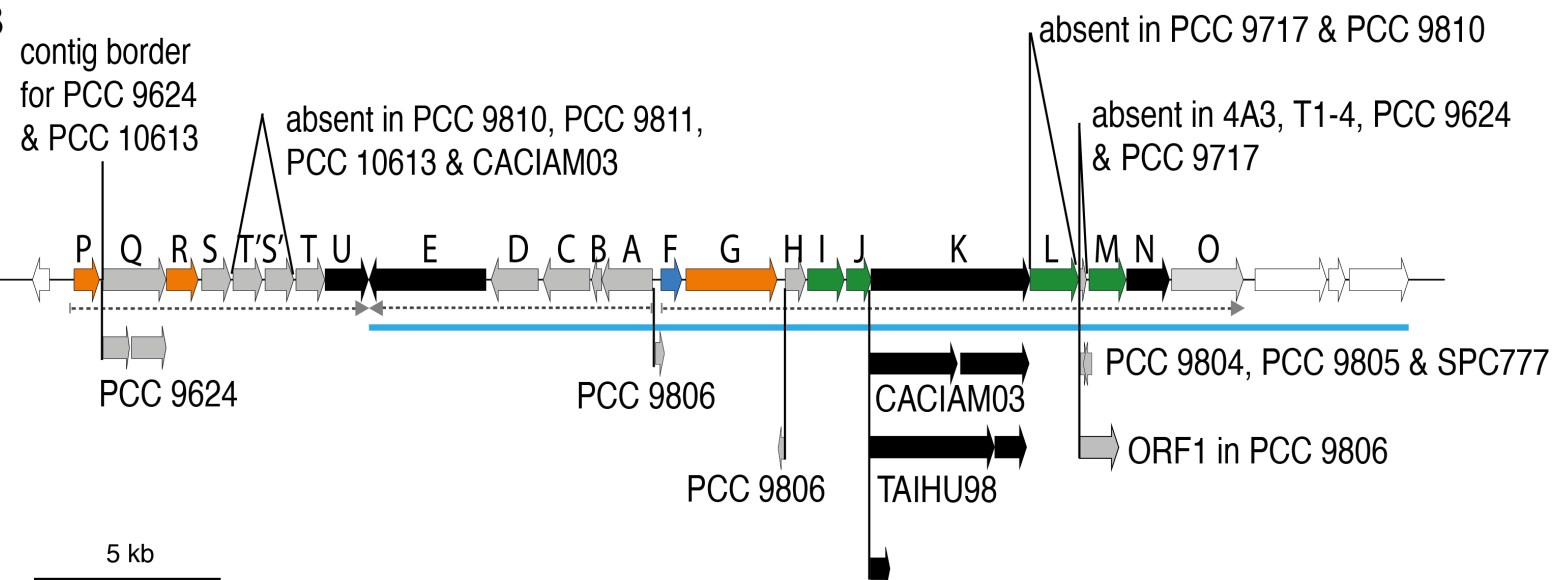

PCC 10613 
Figure 2. Molecular network of AGD (A) and of MGD with MGA (B). Characterized structural variants are indicated as red-colored nodes and new congeners charactized in this study are highlighted in green. Diversity and distribution of AGD and MGD variants for each strain. Details of $\mathrm{MN}(+), \mathrm{MN}(-)$, and the AGD and MGD networks are presented in Figures S1-S3.

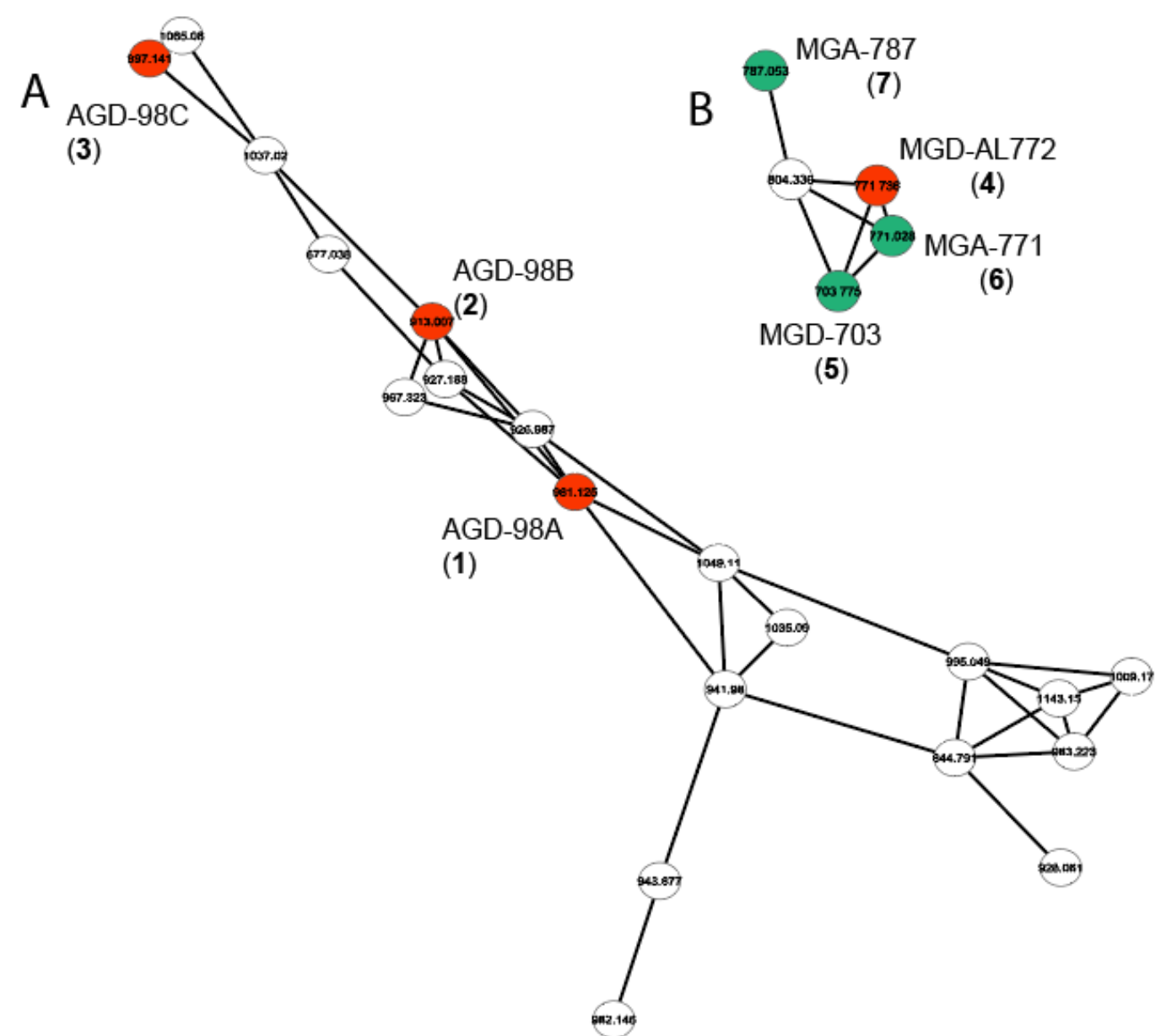

\begin{tabular}{|c|c|c|c|c|c|c|c|c|c|c|c|}
\hline Parent Mass & & & & & & & & & & & \\
\hline$m / 2(M-H)$ & NIES_98 & \begin{tabular}{|l|} 
PCC 9624 \\
\end{tabular} & PCC 9717 & PCC 9804 & PCC 9805 & PCC 9806 & PCC 9810 & PCC 9811 & PCC 10613 & $T 1-4$ & Assignment \\
\hline 667.038 & & & & & & & & & & & \\
\hline 844.791 & & & & & & & & & & & \\
\hline 913.007 & & & & & & & & & & & AGD-98B (2) \\
\hline 926.987 & & & & & & & & & & & \\
\hline 927.186 & & & & & & & & & & & \\
\hline 928.061 & & & & & & & & & & & \\
\hline 941.98 & & & & & & & & & & & \\
\hline 949.877 & & & & & & & & & & & \\
\hline 967.323 & & & & & & & & & & & \\
\hline 981.125 & & & & & & & & & & & AGD-98A (1) \\
\hline 982.146 & & & & & & & & & & & \\
\hline 983.223 & & & & & & & & & & & \\
\hline 995.049 & & & & & & & & & & & \\
\hline \begin{tabular}{|l|}
997.141 \\
\end{tabular} & & & & & & & & & & & AGD- $98 \mathrm{C}$ (3) \\
\hline \begin{tabular}{|l|}
009.17 \\
\end{tabular} & & & & & & & & & & & \\
\hline 1035.09 & & & & & & & & & & & \\
\hline 1037.02 & & & & & & & & & & & \\
\hline 1049.11 & & & & & & & & & & & \\
\hline 1065.08 & & & & & & & & & & & \\
\hline 1143.15 & & & & & & & & & & & \\
\hline 703.775 & & & & & & & & & & & MGD-704 (5) \\
\hline \begin{tabular}{|l|}
771.028 \\
\end{tabular} & & & & & & & & & & & MGA-771 (6) \\
\hline 771.736 & & & & & & & & & & & MGD-AL772 (4) \\
\hline 787.053 & & & & & & & & & & & MGA-787 (7) \\
\hline 804.336 & & & & & & & & & & & \\
\hline
\end{tabular}


Figure 5. Distribution of the known and unknown BGCs in the frame of the phylogeny of the 23 Microcystis genomes based on maximum likelihood tree built upon 586 marker genes. The known BGCs are involved in the synthesis of aeruginoguanidine (AGD) and microguanidine (MGD) and/or microguanidine amide (MGA) only, of microcystin, of cyanobactins including aeruginosamide, of aeruginosin, of microviridin, of cyanopeptolin, of anabaenopeptin including ferintoic acid, and of microginin. One BGC only predicted in one strain is involved in synthesis of puwainaphycin. ${ }^{12}$ The numbers indicate the unknown BGCs detected in the genome; the abbreviations in parenthesis after the name of the strain indicate its origin (Table S2).

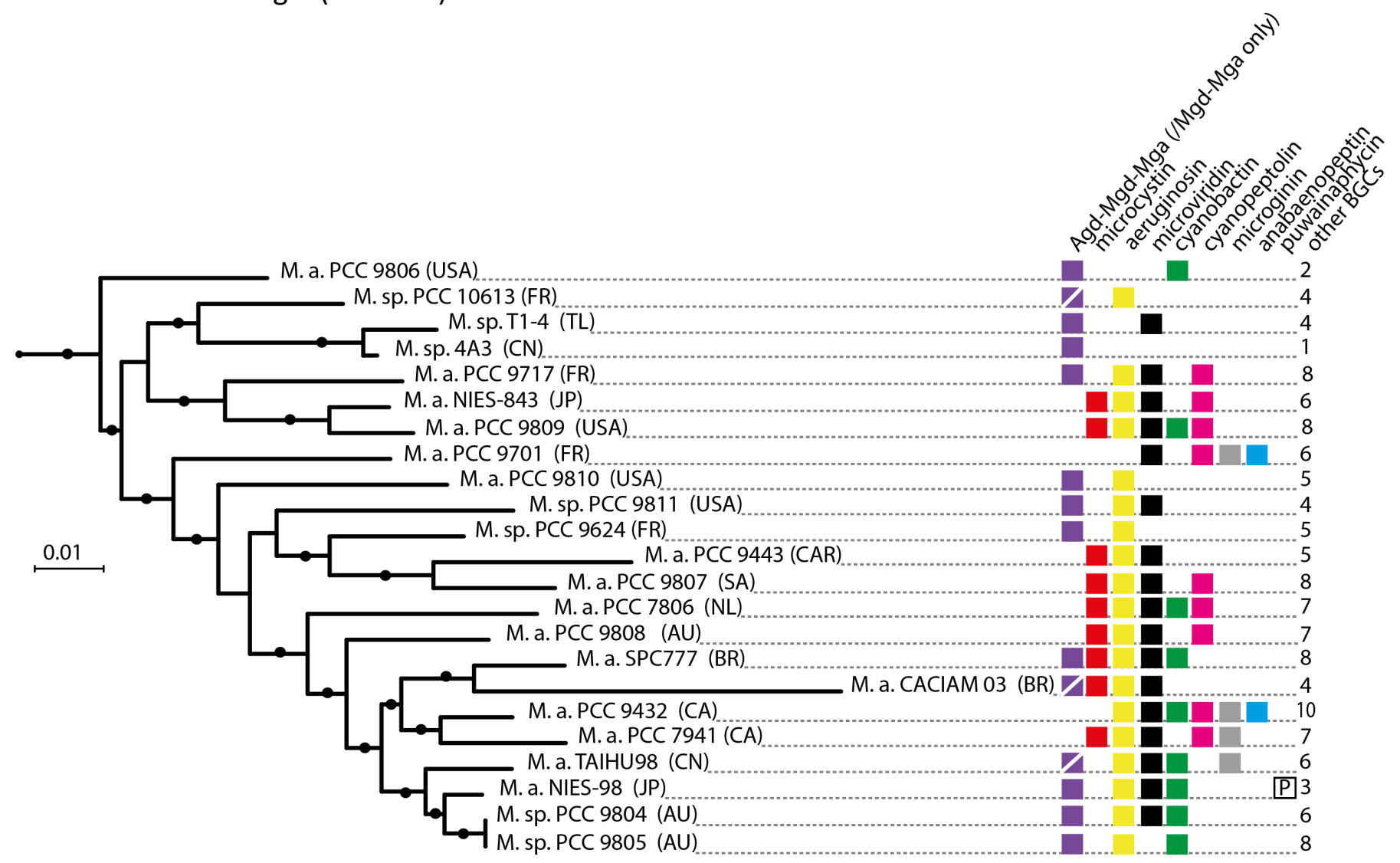


<smiles>CC(C)=CCC/C(C)=C\CN(CCCC(C(=O)OC(C)C(OS(=O)(=O)O)c1ccc(OS(=O)(=O)O)c(COS(=O)(=O)O)c1)[N+](C)(C)C)C(=N)N</smiles>

\section{Microguanidine AL772 (4)}<smiles>CC(C)=CCN(CCCC(C(=O)OC(C)C(OS(=O)(=O)O)c1ccc(OS(=O)(=O)O)c(COS(=O)(=O)O)c1)[N+](C)(C)C)C(=N)N</smiles><smiles>CC(C)=CCC/C(C)=C\CN(CCCC(C(=O)NC(C)C(OS(=O)(=O)O)c1ccc(OS(=O)(=O)O)c(COS(=O)(=O)O)c1)[N+](C)(C)C)C(=N)N</smiles><smiles>C=C(C)C(O)CC/C(C)=C/CN(CCCC(C(=O)NC(C)C(OS(=O)(=O)O)c1ccc(OS(=O)(=O)O)c(COS(=O)(=O)O)c1)[N+](C)(C)C)C(=N)N</smiles>

Figure 3. Microguanidine and microguanidine amide variants detected in strains containing the AGD biosynthetic gene cluster. The MGD depsipeptides contain ester-bonds, while the peptidic MGAs contain amide-bonds in their structures. Details of the high-resolution MS data of MGA and MGD are presented in Table S3.

$63 \times 94 \mathrm{~mm}(300 \times 300 \mathrm{DPI})$ 
Figure 4. Proposed Hphpa and Hphpol biosynthesis. 3-hydroxy-m-hydroxymethyl-p-hydroxyphenylpyruvate is synthesized as a precursor of both $\mathrm{Hphpa}$ and $\mathrm{Hphpol}$ via several steps from isochorismate. The resulting intermediate is further transformed by either an aminotransferase (AgdL) or a reductase (AgdN or $U$ ) to yield Hphpa or Hphpol, respectively and further transferred to the free-standing PCP (AgdB) after adenylation by AgdA. The AgdL enzyme for which natural mutants were identified in the course of this study is highlighted in red.

$130 \times 74 \mathrm{~mm}(300 \times 300 \mathrm{DPI})$ 


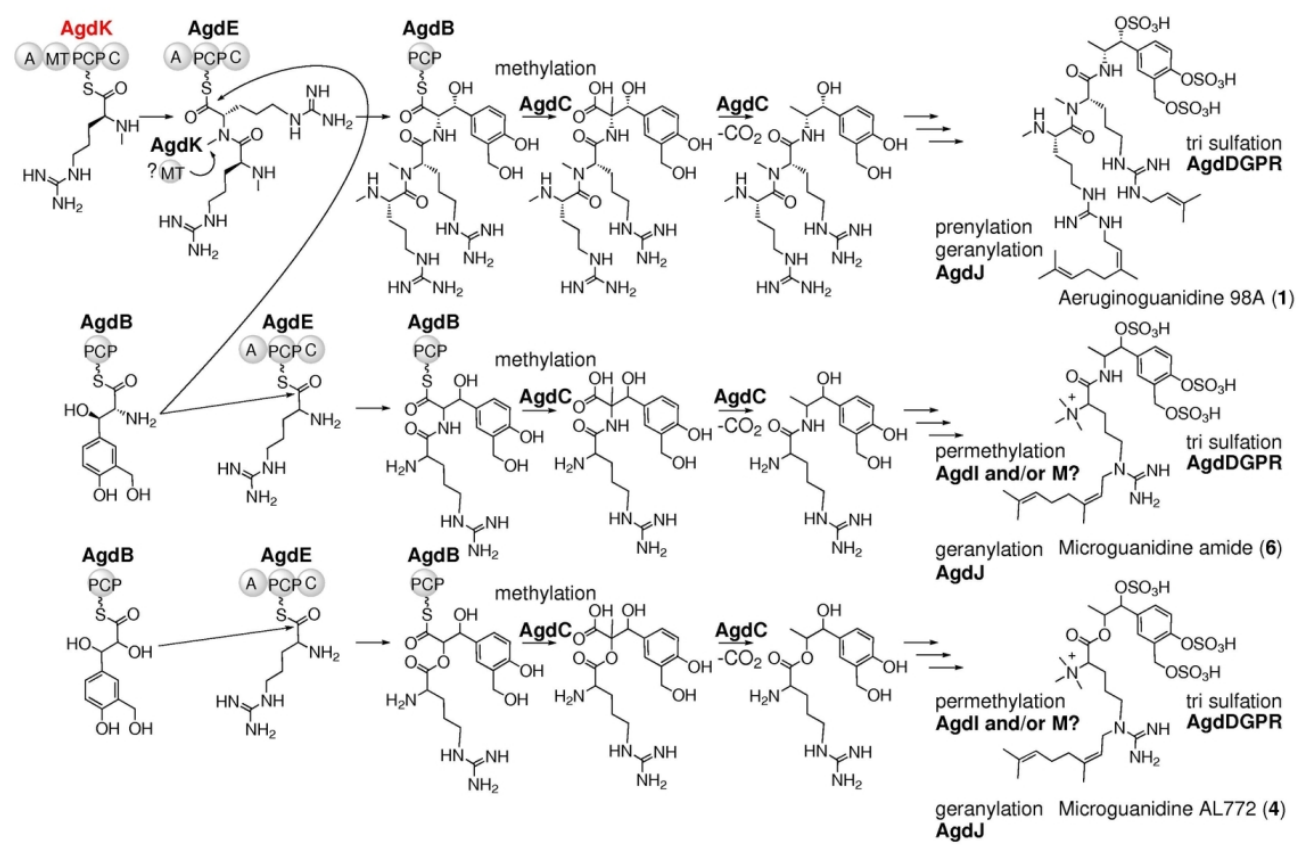

Figure 6. Proposed AGD, MGA and MGD biosynthetic pathways. Top line; AGD biosynthesis route: Hphpa which is linked to the free-standing PCP AgdB is transferred to AgdE and condensed with the dipeptide, which is derived from AgdK and $\mathrm{E}$. The thioester-tethered intermediate is methylated by a radical SAM enzyme (AgdC) followed by decarboxylation and released from the enzyme. The resulting molecule is further modified by sulfation and farnesylation. Middle line: MGA (6 and 7) biosynthesis route, almost the same pathway as AGD biosynthesis, but only AgdE is used and the a-amino group of Arg is permethylated by AgdI or M. Bottom line; MGD (4 and 5) biosynthesis route, almost the same pathway as MGAs, but using Hphpol as the intermediate instead of Hphpa. The enzyme AgdK for which a natural mutant was identified in the course of this study is highlighted in red. 


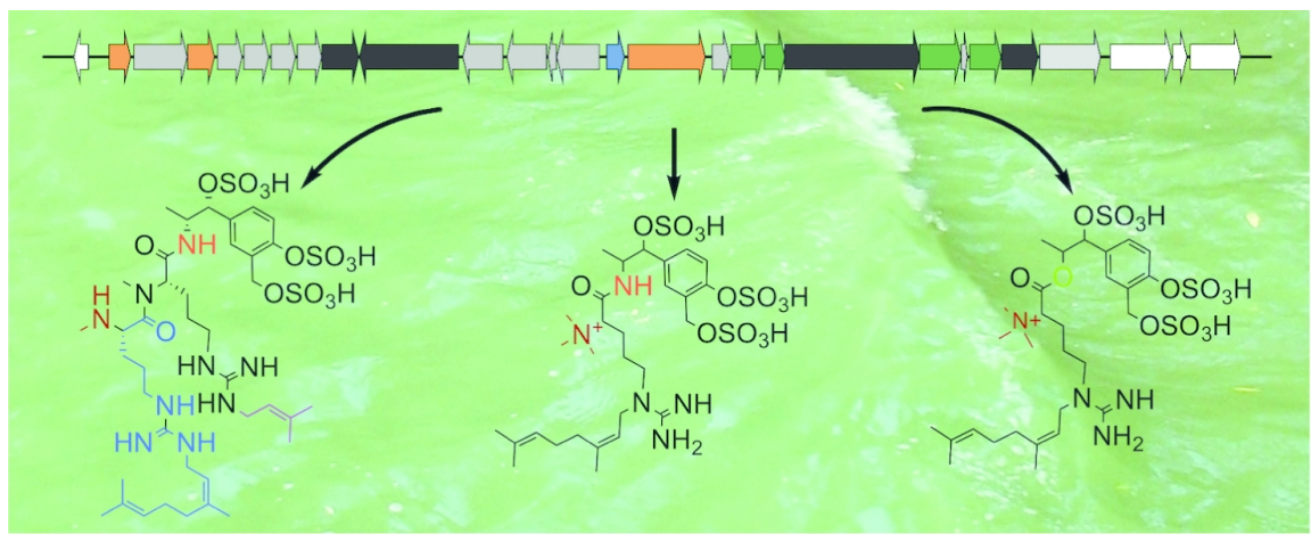

A Unique Biosynthetic Pathway in Bloom-Forming Microcystis Jointly Assembles Cytotoxic Aeruginoguanidines and Microguanidines 